\title{
An approach to compare tension chording concepts by using combined multibody and finite element simulation
}

\author{
Eike Uttich ${ }^{1 *}$, Beate Bender ${ }^{1}$ \\ ${ }^{1}$ Ruhr-University Bochum \\ * Corresponding author: \\ Eike Uttich \\ Chair of product development \\ Ruhr University Bochum \\ D-44780 Bochum \\ Phone: 0234/3226008 \\ Mail: uttich@lpe.rub.de
}

\begin{abstract}
The tension chording principle is a biomimetic lightweight design principle for kinematic chains. A great number of conceivable actuation concepts exist for specific applications that use the tension chording principle. Their effect on the system weight cannot yet be evaluated without calculating structural stress and movement. When comparing the effects on system weight of different actuation concepts, modelling the concepts can be timeconsuming. We present an approach that uses a modeling system combining multibody and finite element simulation. Thus, stress and movement are calculated in the same model. We successfully test the approach for a simple system.
\end{abstract}

\section{Keywords}

Biomimetics, Lightweight design, Simulation, Tension chording principle 


\section{Introduction}

Lightweight design (LWD) is a design philosophy that aims at reducing weight while retaining the other product properties. It is a strategy to find a system of minimum weight, which fulfills all desired functions and technical requirements [1]. Research in LWD involves the search for and utilization of new LWD principles. One field of research is system LWD, in which researchers search for principles that use interdependencies in the entire system to reduce the overall system weight. A place to search for new principles is nature, as it invented various systems that contain potential LWD principles [1].

Biomimetic methods support the transfer of LWD principles observed in nature to technical applications [2]. If the transfer process is initiated by technology as a method to synthesize solutions, it is called "Technology-Pull". "Biology-Push" names the other case, in which the transfer process is initiated by the discovery of a principle in biology and is ended with its application to a technical problem [2]. In the past, research groups transferred lightweight design principles from the human arm e.g. to robots [3]. We have run a "Biology-Push" process by using biomimetic methods on the actuation of the human arm to describe a new system LWD principle called "tension chording principle" and to transfer it to a robot $[4,5]$.

"Tension chording principle" is a principle to reduce stress in the structure by controlling the load distribution in the structure. It is a system LWD principle, because interdependencies between actuation and structure are utilized in the principle. Controlled load distribution can reduce the demands on structural robustness. Consequently, the weight of the structure can be decreased. However, adding load distribution control to the functions of the actuation system can require an increase of weight in the actuation system. Additionally, the principle can cause the use of different actuation types with different weight, e.g. by shifting from engines in joints to hydraulic cylinders or belt drives. Consequently, some solutions that fulfill the principle have a higher weight than other solutions with or without the principle. The challenge in the application of the system LWD principle is to find the solution with the lowest weight for the overall system rather than reducing weight in each subsystem.

Our long-term research goal is to develop tools that help to decide which solution is applicable for specific boundary conditions without the need of extensive simulation. We plan to reach this goal by investigating a number of different tension chording principle concepts and solutions. For the investigation, we need to determine the resulting weight for various solutions. To determine the structure weight, we are planning to use the optimization process for mechatronic systems [1] in form of a simulation workflow. To reduce the engineering time spent for executing the optimization, we aim for a high degree of automation for the simulation workflow. In this paper, we are applying the first step of the simulation workflow, which is load calculation, for a tension chording solution to test the use of a software tool called ARTISYNTH for our purposes.

First, we present the simulation workflow and the tension chording principle (section 2). Afterwards, we motivate the use of combined multibody and finite element simulation in the load calculation. To do this, we adapt the workflow for the tension chording principle (section 3). Afterwards, we present an adapted workflow and the general concept of how we realize the load calculation with simulation software (section 4). Subsequently, we apply the adapted load calculation to a minimal example of a system with tension chording principle and discuss influencing factors for the approach (section 5). The paper ends with remarks on further works that are possible to extend the simulation model or necessary to realize the workflow in a mostly automated manner (section 6). 


\section{Background}

This section contains a short overview of system LWD and the tension chording principle. Extensive information on lightweight design are available e.g. in [1], [6], and [7]. We use the definitions on lightweight design strategies as introduced in [1] in this paper. Extensive information on the tension chording principle and the virtual prototype developed in our lab that includes the tension chording principle are available in [5].

\subsection{System lightweight design}

System lightweight design is a design strategy that aims at minimizing the overall system mass and inertia. The system is optimized holistically while considering all interdependencies and relations in the system. Additionally, general technical and economical boundary conditions are considered in the optimization. By asking which elements are necessary to the desired function, system lightweight design can lead to new system concepts. In these designs, functions are integrated from several to a single system element (functional integration) or separated from a single to several system elements (separation of functions). In both cases, the aim is to reduce the overall system weight [1].

Inside a system, isolated lightweight design strategies like the application of lightweight materials or lightweight structure design contribute to reducing the overall system weight. Using lightweight materials aims at generating the product with the lightest possible material for the given boundary conditions. Using lightweight structures aims at finding the lightest structure for the given boundary conditions and load distribution. If put in a hierarchic interdependence, the strategy lightweight structures encloses lightweight material and is enclosed by system lightweight design. Lightweight structures depend on the material that the lightweight material strategy influences. It also depends on the design space that system lightweight design influences. The material depends on the boundary conditions set by the surrounding system elements, which system lightweight design influences by changing the arrangement of system elements [1].

Technical systems and their interdependencies are complex and therefore difficult to solve by analytical approaches. Literature presents numerical approaches that combine Finite Element Analysis (FEA), multibody simulation (MBS) and simulation tools like MATLAB/Simulink to optimize mechatronic systems [1]. We describe an approach from literature with this aim in section 2.2.

\subsection{A simulation workflow for system lightweight design}

System lightweight design exploits interdependencies between system elements to reduce the overall weight of the system which are often complex. To handle the interdependency between structure mass and the system design concepts, literature offers the workflow shown in Figure 1 [1]. Topology optimization is used in this workflow to determine the mass of the structures that is required to fulfill an optimization criterion. In our case, this is the stiffness of the structure. We optimize it by changing the mass until the stiffness is just as high as needed to support the loads applied by the actuators and external loads. Control of movement has an influence on the loads on the structure. For example, if a starting point and an end point are defined but the path and time to get from A to B are free to choose, loads can vary depending on the path chosen by the control system. The longer it takes to move from start to end, the smaller the impact of the dynamic loads and the higher the influence of the static loads. As dynamic loads add to the static loads, reducing the former loads leads to a lower demand for stiffness of the structure. 


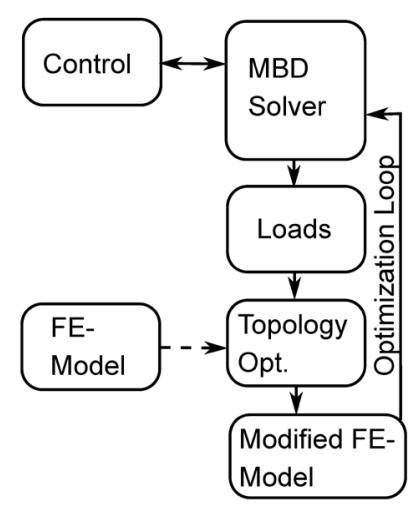

Figure 1: A simulation workflow to account for interdependencies between lightweight design strategies in the calculation of structure mass. Modified representation of Figure 5.9 (p. 126) in [1].

Loads to be used for topology optimization are preferably calculated automatically [1]. In the optimization process, this becomes possible by two steps: One, simulating the desired actions with a multibody dynamics (MBD) solver coupled to a control system. Two, automatically choosing e.g. load maxima calculated in the simulation.

\subsection{Tension chording principle}

The human musculoskeletal system contains several subsystems with interdepending lightweight design solutions [8]. One part of the system is the human arm. In this paper, we focus on the actuation system of the human arm. Its actuation includes the muscles and the brain controlling the muscular activity with the help of nerves that measure the state of the arm or stimulate the muscles [9]. Concerning LWD principles from the actuation system of the arm, we focus on two principles: (1) guy-wire support effect and (2) the tension chording principle.

When muscles move the arm, they work as support similar to guy-wires that support guyed masts (e.g. antennas). Figure 2 illustrates the support effect of guy-wire for a column under off-centered load. Guy-wires and muscles reduce the bending in the structures, which allows for lower stiffness of the bones (or antennas). In other words, the muscles and guy-wires take a part of the tension out of their systems by transforming the bending into compression loads.

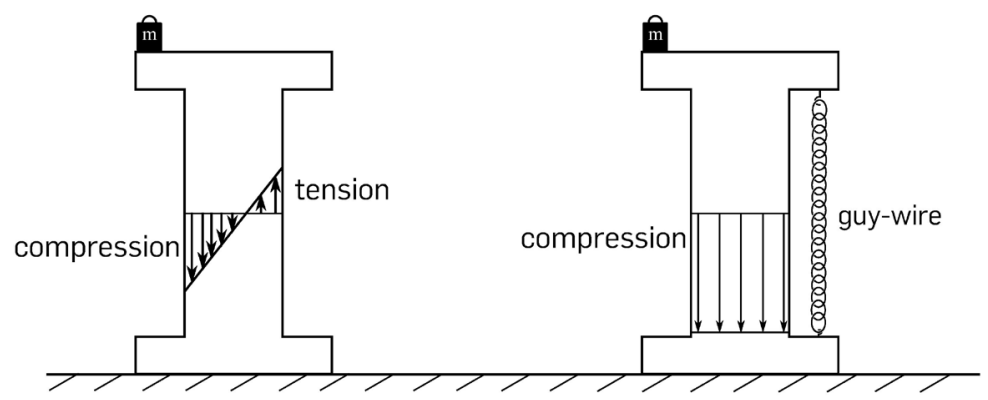

Figure 2: The effect of a guy-wire for a column under off-centered load. Modified representation of Figure 7 (p. 404) in [10]

The tension chording principle is based on the observation that there are more muscles available than needed to move the arm, i.e. some muscles are redundant. Figure 3 illustrates the tension chording principle for a system analog to the human arm and for the task of holding a weight in a static position. We included eight force effectors to represent muscles that the brain can use to fulfill the task. Only one muscle for each segment is needed in this simple example to define the position of the segments. As bending highly influences the stress in the structure and is influenced by the muscles, the figure contains the resulting bending in the structure for two cases. In both cases, the bending in one of the segments (upper arm or lower 
arm) is low. At the same time, bending is high in the other segment. This results in a low mass for the segment with low bending and a high mass for the segment. The human brain's task is to find a muscle activation where the sum of bending is minimal. Transferred to technology, the tasks of the brain become an optimization problem of control.
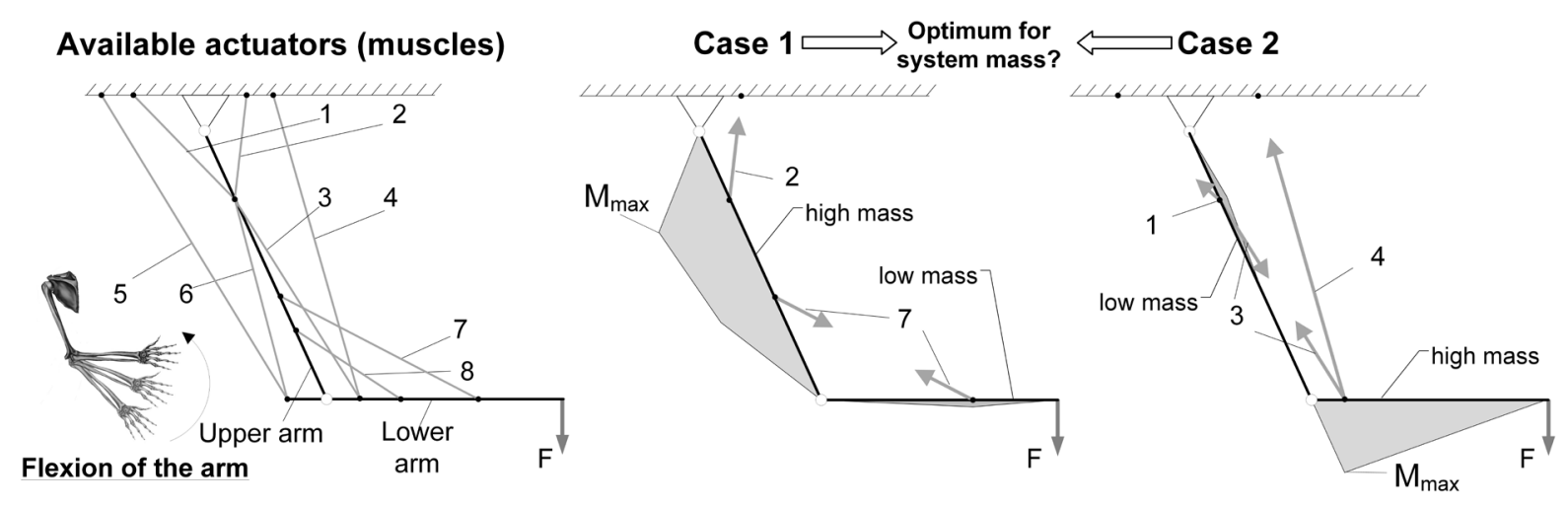

Figure 3: General concept of tension chording and two extreme cases. Modified representation of Figure 2 in [8]

In technical applications, the tension chording principle is a system LWD principle for actuation concepts that includes both the actuation concept and actuation control. Using the principle for a technical system can cause fundamental changes in the actuation concept of the system [5]. The main aspect of the principle is the functional integration of "determine movement" and "control component stress" in the actuation system. Guy-wires and actuators (muscles in nature) both determine the movement, but guy-wires are limited to retain the structure in a static position. Control (the brain in nature) utilizes the guy-wire support effect of the actuators to control component stress. The minimum number of actuators required for the tension chording principle is two. However, the more actuators are available in different positions, the more influence has control on component stress. Controlling component stress decreases demands on robustness of the components. Component form can be adapted to decreased demands on its robustness by reducing its weight.

A factor that influences the effect of the tension chording principle is the actuation type. Several actuators offer the guy-wire support effect, e.g. hydraulic and pneumatic cylinders, artificial muscles, belt drives and linear electromagnetic actuators. Compared with each other, the actuator types differ in several aspects, e.g. their power range, their mass to power ratio, and their range of motion. Compared to joint engines, actuators with guy-wire support effect cause less bending in the structure. The actuator type influences the total system weight.

In other publications, the tension chording principle is described as a principle to reduce bending in structures by using redundant muscle and tendon forces $[8,5]$. We introduce a refined definition in this paper. The purpose of the refined definition is to emphasize that the tension chording principle depends on and utilizes the LWD principle guy-wire support effect. Actuators with guy-wire support effect can be used without using the tension chording principle. However, the tension chording principle is not applicable without redundant actuators that have a guy-wire support effect.

To account for the interdependency of the tension chording principle to lightweight structure design, we developed a simulation workflow [5] which is similar to the more general workflow [1] presented in section 2.2. For control and MBD, we use MATLAB in combination with a mechanical model of the system based on beam theory. Topology optimization is executed in ANSYS. A separate model is generated in ANSYS for the topology optimization. Force sets are exported from MATLAB and manually inserted into the ANSYS model. The results of the topology optimization are reworked and the resulting mass of the structure is used in MATLAB for the next iteration step. This workflow is not automated yet. 


\section{Research problem and goal}

Our research addresses the technical application of the tension chording principle. It is a system LWD principle with interdependence to other LWD principles. We use an existing simulation workflow that helps accounting for the interdependence for the tension chording principle. The simulation workflow allows us to evaluate the structure mass of each tension chording solution individually. It is executed by exporting data from one simulation program and importing them in another program. Currently, the workflow requires taking action several times during its execution in terms of operating the different programs used in the workflow. We noticed that the number of iterations required to converge against a structural mass is low. Therefore, our long-term research goal is to automate the process from calculating the loads to using the topology optimization. In this case, the workflow only requires taking action when a new iteration starts.

In multibody systems, we connect body parts with joints and apply forces. The multibody simulation software calculates the mathematical equations of the assembled system in the background. We consider the assembly of the system as high level modeling, while the mathematical equations are low-level modelling. For tension chording control, we currently use mathematical equations based on beam theory for optimization of stress and movement. This low-level modeling is necessary as multibody dynamics is not designed to provide information on the stress in structures required for tension chording control. Currently, we express the mathematical equations as functions in MATLAB for control. We use CAD for design and FEM in ANSYS for topology optimization. However, to validate the equations for several concepts and modelling them in several systems can be a time-consuming task. As we plan to investigate numerous concepts, we need to save time in the modelling process. We can save time by reducing the number of models required to simulate a single concept and by avoiding the use of mathematical equations to model the system for control. How can we adapt the LWD optimization process for investigating tension chording concepts? How can we realize tension chording control in this process with high level modelling? The research goal of this paper is to test a simulation software called ARTISYNTH in combination with MATLAB for realizing tension chording control by only using high level modeling for a minimal example of a tension chording system.

\section{Methods and approach}

Tension chording control requires information on the stress or bending in the structures. Currently, the control algorithm simulates the effect of different actuation forces on the load of the structure for short time intervals. The algorithm iterates to the one that fits best to the criteria given beforehand. Those criteria can be e.g. the integral of bending over the length of a beam, the sum of stresses in the structure, the maximum stress in the structure, or multiple criteria. MATLAB provides optimization functions for such problems.

To calculate the stresses needed for control, we add Finite Element Analysis (FEA) to the MBD-control-system as indicated in blue color in Figure 4. To solve both MBD and FEA with the same model, we use ARTISYNTH (www.artisynth.org) in this paper. ARTISYNTH is an open source 3D mechanical modeling system that combines multibody and finite element simulation [11]. Instead of using an FEA program and a MBS program in cosimulation, ARTISYNTH includes both methods in one simulation environment. It offers an interface to MATLAB by using the MATLAB-Java-Interface, which allows changing and executing the system created in Java with program code in MATLAB.

ARTISYNTH models are written in Java classes that extend the generic ARTISYNTH root model. By extending the generic root model, all basic ARTISYNTH functions are usable inside the model created for a specific purpose, e.g. FEM bodies, joints, and force effectors. The defining parameters to describe a model at a simulation time t completely are called state. 
States can be saves and jumped to, which allows repeating a part of a simulation with changed boundary conditions. Our approach is based on the FEA capabilities of the MBD system and the possibility to save states and jump back to them.

We realize model control in MATLAB as an optimization process to minimize the stress in the structure and the position error. In Figure 4, we illustrate the optimization process for one time step. The optimization process for one time step consists of five steps, which we categorize into preparation, iteration and post processing. To prepare the optimization, MATLAB tells ARTISYNTH to save the current state of the model (step 1: Save State). This enables MATLAB to jump back to this state during iteration. In iteration phase, MATLAB sets actuation values to predefined start values or values different from the previous time the values were set (step 2: Set Values). It tells ARTISYNTH to execute the simulation with these constant actuation values for the time step (step 3: Execute). MATLAB reads the resulting position and stress from ARTISYNTH and jumps back to the saved state (step 4: Read Results). During the optimization, MATLAB reruns steps 2 to 4 as an iteration until it finds a minimum for a cost function of position error and the overall stress in the structure. With a minimum found, post processing of the optimization starts. MATLAB tells ARTISYNTH to run the simulation once more with the optimal input values for the time step. As a result, ARTISYNTH arrives at a new state and time while the actuation fulfills the optimization criteria (step 5: Advance). MATLAB saves the input values in a result vector that we use to simulate the results for the total simulation time without iterations. Afterwards, MATLAB restarts the process by saving the new state for the iteration of actuation forces in the next time step.

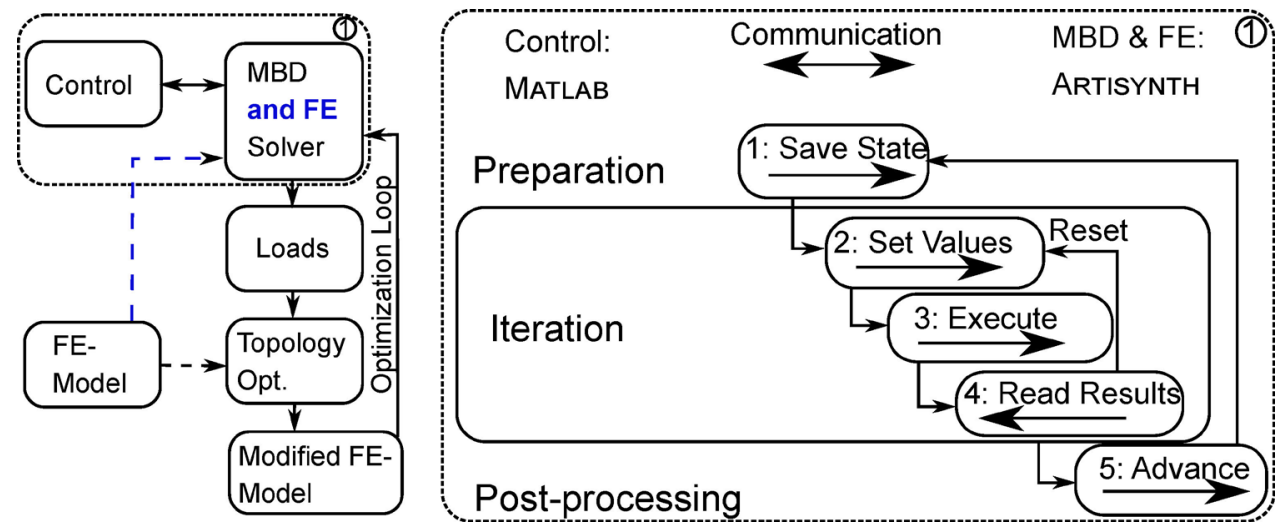

Figure 4: Extension of the simulation workflow to account for the interdependencies of lightweight design strategies (left). General steps of an optimization for one time step inside this workflow (right). The arrows inside each step indicate the direction of communication, e.g. an arrow to the right means that MATLAB send a command to ARTISYNTH

We test the described coupling of ARTISYNTH and MATLAB with a minimal example of tension chording. The simplest example of tension chording is a beam that a revolute joint connects to a world frame and with two actuation forces. With one degree of freedom, two actuation forces are enough to create the redundancy needed for the tension chording principle. For the minimal example, we do not include actuation models to calculate the actuation forces. Factory methods of ARTISYNTH create and mesh the beam structure. We set the mesh to hexahedron linear and a coarse size of 6 times 3 times 3 elements for the beam. This leads to 54 elements in the mesh over a length of $50 \mathrm{~cm}$, a width of $20 \mathrm{~cm}$ and a height of $10 \mathrm{~cm}$. The density of the beam material is set in the interval of the density of steel, resulting in a weight of $78.5 \mathrm{~kg}$ for the beam. Additionally, we include a small amount of rotary damping to avoid infinite swinging of the system. Figure 5 presents the system with the two force effectors (actuators). MATLAB controls the actuators. 


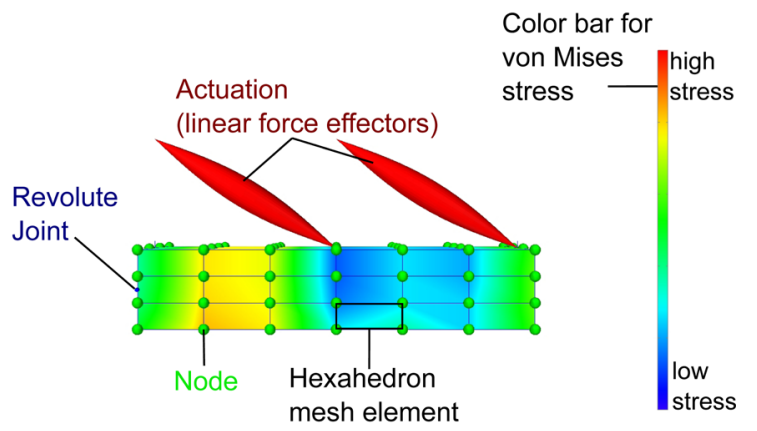

Figure 5: ARTISYNTH-model with two force effectors positioned in the center of mass and at the end of the beam. The coloring of the beam results from numeric errors of a maximum size of $1 \mathrm{e}-5$ for the displayed beam in initial state

During the simulation of the minimal example, the optimization goal for control in MATLAB is to minimize the position error and stress in the beam. To realize control, we use the optimization algorithm PatternSearch, which is a function in MATLAB. The optimization follows two objectives: Minimize the position error and minimize the sum of stresses for all nodes. We define a cost function for those criteria. It is a weighted sum of position error and sum of stresses, which returns one value to the optimization algorithm. Setting the interval of position error as a constraint for the optimization and the stress as optimization objective is simpler, as the weights do not necessarily need to be set. However, this approach leads to longer calculation time than the approach with a cost function. We set the simulation duration to 3 seconds and the time step to 0.1 seconds. The target position is set to the horizontal position of the beam for the whole simulation. After one second, a load in vertical direction pointing to the ground is added at the end of the beam that is opposing the joint. The load increases over time from 0 to $1000 \mathrm{~N}$. The maximum value of the load is set to be larger than the weight of the beam at the end of the simulation. We add this load to observe the effect of a changing force on control of force distribution and the resulting stress in the beam. By adding the force after one second, we have the chance to observe the ability of the control to stabilize the system in the target position.

\section{Results and discussion}

The realization of the MATLAB-ARTISYNTH coupling worked as intended. We simulated the scenario described and found that control in MATLAB was capable to minimize both position error and the von Mises stress in the structure calculated in ARTISYNTH. However, we find that the actuation force distribution sometimes changes quickly from one time step to the next. This is due to three reasons: (1) missing constraints for control concerning the change of actuation activation; (2) a missing actuation model in the setup we used; (3) the optimization algorithm sometimes converging to a local instead of a global minimum. A consequence of reason (2) is that changes in the actuation had a direct effect on the system without a delay. The system would therefore benefit from including a delay in an actuation model in favor of more realistic results.

We used systems with three different actuation to see the effect of tension chording on the stress in the structure. Actuation type 1 is a joint engine imposing a torque around the rotation axis of the revolute joint on the structure and thereby moving it (1, "Joint Engine"). Actuation types 2 and 3 are forces located at the end of the beam (2, "Actuation End") or over the center of mass ( 3 , "Actuation Middle"). The system fulfilling the tension chording principle is actuation type 4 (4, "Redundant Actuation"). It is a combination of actuation type 2 and 3 . We can already compare the different actuation concepts by investigating the maximum and the overall stress in the structure. Table 1 presents the stress at simulation time 0.9 and 2.1 , i.e. shortly before the external load is applied at the end of the beam and after the external load reached its 
maximum. When comparing the stresses, we find that the system with joint engine (1) has the highest maximum stress and sum of stresses for all nodes, which is what we expected. The redundant actuation (4) has the lowest values for $2.1 \mathrm{~s}$ for maximum and sum of stress, but for $0.9 \mathrm{~s}$ it has higher values than "Actuation Middle" (3). It appears that the optimization did not find the global optimum in this case. The ability of the optimization to find the global optimum is a crucial factor that influences the resulting stresses and the comparison of the actuation concepts.

Table 1: Maximum stress and sum of stresses at all nodes in MPa for simulation times $0.9 \mathrm{~s}$ (body load) and 2.1s (body load and $1000 \mathrm{~N}$ external load). We display the beams illustrating the actuation concepts in their initial state

\begin{tabular}{|lr|ll|lc|}
\hline \multirow{2}{*}{ Actuation concept } & \multicolumn{2}{|l|}{ Maximum stress [MPa] } & \multicolumn{2}{l|}{ Sum of stresses at all nodes [MPa] } \\
\cline { 2 - 6 } & $0.9 \mathrm{~s}$ & $2.1 \mathrm{~s}$ & $0.9 \mathrm{~s}$ & $2.1 \mathrm{~s}$ \\
\hline \multirow{2}{*}{ Joint Engine } & 0.264 & 1.049 & 7.771 & 37.519 \\
\hline Actuation Middle & & 0.067 & 0.299 & 2.812 & 14.959 \\
\hline Actuation End & & 0.121 & 0.280 & 5.801 & 11.666 \\
\hline $\begin{array}{l}\text { Redundant } \\
\text { Actuation }\end{array}$ & & 0.086 & 0.226 & 3.689 & 9.150 \\
\hline
\end{tabular}

Another factor influencing the stress results is the mesh. While simulation time is decreased significantly with a coarse mesh size, statements on the actual mass savings according to the stress are unlikely to be reliable and detailed with the chosen resolution. Thus, a decision between certain concepts that require precise information on mass savings is not possible with this simulation. It would require another FEA with a smaller mesh size and a subsequent topology optimization. However, it is possible to get a general idea of the effect that different actuation concepts can have in combination with tension chording. This can reduce the number concepts that require topology optimization and therefore save time.

The approach presented in this paper helps to avoid modelling on a low level in form of mathematical equations. These equations can be used to validate results, but they are not necessary anymore for the design and evaluation of a tension chording system. We could show for a minimal example that the approach can be used to simulate tension chording systems. The stresses calculated with the approach reveal trends concerning the effect of tension chording concepts for LWD: The lower the overall and maximum stresses for the same design space, material and application case, the better the actuation concept concerning its impact on reduction of structural mass. If the stresses calculated for control are accurate for a rough comparison of solutions, some solutions can be excluded before topology optimization. To what extend the stresses used for control are valid and have to be valid for design is to be investigated in future studies.

As the human body is a tension chording system, the approach could be used for forward dynamic simulation of human movement with optimization and control executed in MATLAB. ARTISYNTH is mainly developed to be used for musculoskeletal simulation. Besides the tension chording criterion of minimizing stress of bending in the structure, other criteria, as already used in other simulation software, can be implemented and investigated in MATLAB. 


\section{Summary and future work}

We present an approach to compare tension chording concepts by using a simulation software that combines multibody dynamics and finite element analysis. The combination is necessary for the tension chording principle as it controls stresses in the structure. As it is a system lightweight design principle, it has interdependencies to other lightweight design strategies, e.g. structure lightweight design, and lightweight design material. It also has interdependencies with other system lightweight design principles. The interdependencies hinder the estimation of mass of the overall system for the concept. Instead, they require simulations to calculate the mass. We adapt the load calculation step in a simulation workflow that supports the calculation of system mass [1]. The current approach depends on mathematical equations to calculate the stress in the structure for load calculation. With the approach presented in this paper, stress is calculated by using FEM. We present the approach with a minimal example of a tension chording system. Using the approach, we gained information on the stress in the structure, which we could use to compare the actuation concepts. The results correlate to the resulting mass of the structure, but they depend on several factors e.g. the mesh size. Additionally, determining the mass by topology optimization is an iterative process. Therefore, conclusions on structure mass based on the stress are limited. Consequently, we still need topology optimization to decide between concepts that, based on the stress, appear to have a similar effect on structure mass. However, we can preselect actuation concepts to reduce the amount of concepts where topology optimization is necessary.

Our next goal is to test the approach with a more complex example, where we import the structure rather than creating and using a simple volume with predefined methods. We also plan to investigate the influence of mesh size on simulation results and simulation time. Additionally, we intend to implement the approach in a workflow where MATLAB starts a topology optimization program. In this workflow, the necessary information for the topology optimization are automatically transferred from MATLAB to the topology optimization program. This helps us to develop support for the design of tension chording systems that reduces the necessity of modelling and simulation.

\section{References}

[1] Henning, Frank; Moeller, Elvira: Handbuch Leichtbau. München: Carl Hanser Verlag, 2020.

[2] VDI 6220-1 (Draft), 2019. Biomimetics - Fundamentals, conception and strategy.

[3] Klug, Sebastian et al.: Design and Application of a 3 DOF Bionic Robot Arm. In: AMAM 2005, IIImenau, Germany, September 25-30, 2005.

[4] Gößling, Rainer et al.: Compensation of bending moments as a nature-inspired design principle? In: Design 2014: proceedings of the $13^{\text {th }}$ International Design Conference, Dubrovnik, Croatia, May 19-22, 2014.

[5] Bartz, Marcel; Uttich, Eike; Bender, Beate: Transfer of lightweight design principles from the musculoskeletal system to an engineering context. In: Design science 5 (2019), p. e19_1-e19_21.

[6] Klein, B.: Leichtbau-Konstruktionen: Berechnungsgrundlagen und Gestaltung. Wiesbaden: Springer Vieweg and Springer, 2013.

[7] Degischer, H.-P.; Lüftl, S.: Leichtbau: Prinzipien, Werkstoffauswahl und Fertigungsvarianten. Weinheim: Wiley Online Library, 2009.

[8] Bartz et al: Development of a bioinspired approach for the design of kinematic chains. In: Design 2018: Proceedings of the $15^{\text {th }}$ International Design Conference, Dubrovnik, Croatia, May, 2018.

[9] Uttich, Eike; Bartz, Marcel; Bender, Beate: Factors preventing the use of a lightweight design workflow that is inspired by the human locomotive system. In: Proceedings of the Design Society: International Conference in Engineering Design 1, pp. 2705-2714, Delft, Netherlands, August 5-8, 2019.

[10] Pauwels, F.: Gesammelte Abhandlungen zur funktionellen Anatomie. Berlin, Heidelberg, New York: SpringerVerlag, 1965.

[11] Lloyd, John E.; Stavnes, lan; Fels, Sidney: ArtiSynth: A fast interactive biomechanical modeling toolkit combining multibody and finite element simulation. In: Soft Tissue Biomechanical Modeling for Computer Assisted Surgery (2012), pp. 355-394. 